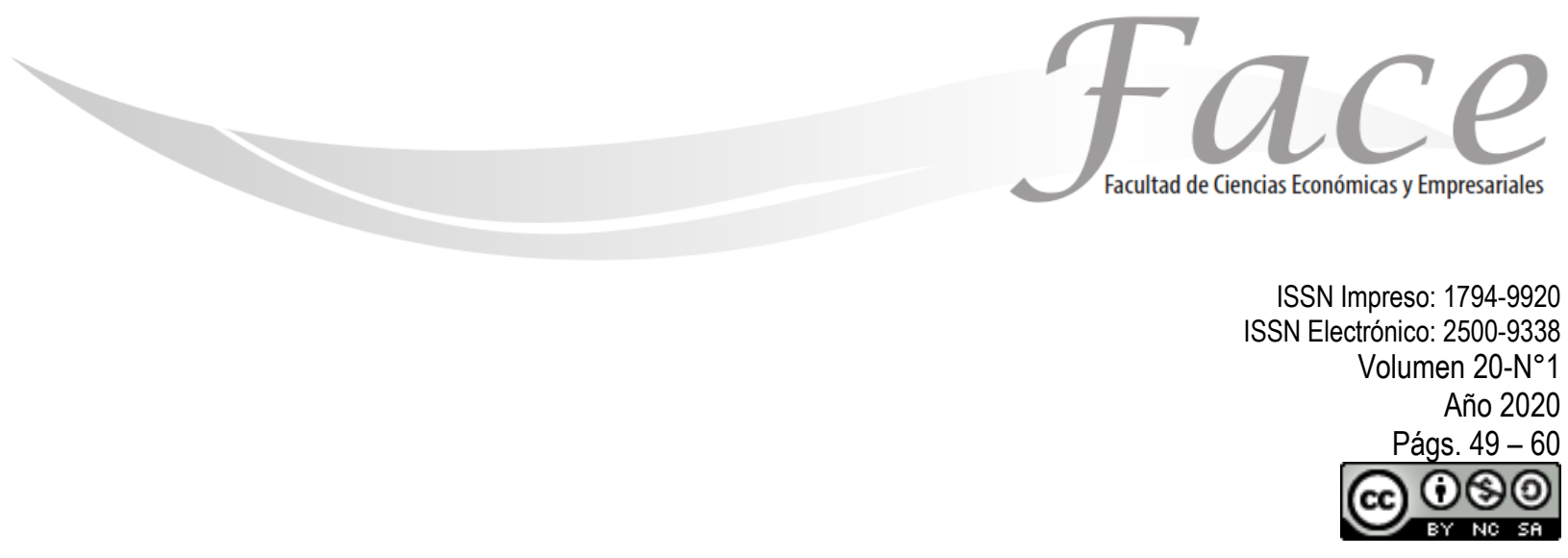

\title{
PÉRDIDAS ECONÓMICAS POR BABOSAS EN FRESA (FRAGARIA × ANANASSA, DUCH) BAJO LAS CONDICIONES DE PAMPLONA, NORTE DE SANTANDER
}

\author{
Leónides Castellanos González * \\ ORCID: http://orcid.org/0000-0001-9285-4879 \\ Junior Serrano Ortiz **
}

Fecha de Recepción: Marzo 30 de 2020

Fecha de Aprobación: Junio 27 de 2020

\section{Resumen:}

El objetivo de la investigación fue estimar las pérdidas económicas causadas por las babosas en fresa bajo las condiciones de Pamplona, Norte de Santander. Se condujo un experimento en un campo cultivado con la variedad Albión. Se evaluaron seis niveles poblacionales de babosas desde 0 (control químico) hasta $>6$ ind./trampa en un diseño completamente al azar. Los niveles de incidencia de frutos dañados por babosas en las parcelas fueron significativos a bajas poblaciones (de 0,1 y 2 ind./trampas) incrementándose hasta $40 \%$. Las pérdidas monetarias por babosas pueden superar las 50000000 COP/ha con índices de hasta 2 babosas/trampa.

Palabras clave: Análisis biliométrico, contabilidad ambiental, base de datos, producción científica, estadística.

* PhD. Facultad de Ciencias Agrarias. Universidad de Pamplona. Km 1 vía Bucaramanga. Contacto: Iclcastell@.gmail.com,

"Ingeniero agrónomo. Facultad de Ciencias Agrarias. Universidad de Pamplona. Km 1 vía Bucaramanga. Contacto: jakson1@outlook.es

El presente artículo es un producto del proyecto: Inventario, incidencia y alternativas para el control de los moluscos plagas en cultivos de importancia económica en el municipio de Pamplona, Norte de Santander. El mismo pertenece a la Convocatoria interna Banco de proyectos 2017 de la Universidad de Pamplona con Código: 400-156.012-006 (GA312-BP-2017) Fecha de inicio:13/02/2018, Fecha de fin:13/08. También forma parte de la tesis de grado del ingeniero agrónomo Junior Serrano Ortiz. 


\title{
ECONOMIC LOSSES DUE TO STRAWBERRY SLUGS (FRAGARIA × ANANASSA, DUCH) UNDER THE CONDITIONS OF PAMPLONA, NORTH OF SANTANDER
}

\begin{abstract}
:
The aim of the investigation was to estimate the economic losses caused by strawberry slugs under Pamplona conditions, North of Santander. An experiment was carried out in a field cultivated with the Albión variety. Six population levels of slugs were evaluated from 0 (chemical control) to $>6$ ind./trap in a completely randomized design. The levels of incidence of fruits damaged by slugs in the plots were significant at low populations (from 0.1 and 2 ind./trap) increasing up to $40 \%$. The monetary losses due to slugs can exceed $50,000,000 \mathrm{COP}$ / ha with indexes of up to 2 slugs / trap.
\end{abstract}

Keywords: mollusks, estimation, damage, harvest

\section{PERDAS ECONÔMICAS DEVIDO A LESMAS DE MORANGO (FRAGARIA × ANANASSA, DUCH) SOB AS CONDIÇÕES DE PAMPLONA, NORTE DE SANTANDER}

\begin{abstract}
Resumo:
O objetivo da investigação foi estimar as perdas econômicas causadas por lesmas de morango nas condições de Pamplona, Norte de Santander. Um experimento foi conduzido em um campo cultivado com a variedade Albion. Seis níveis de população de lesmas foram avaliados de 0 (controle químico) a> 6 ind./ armadilha em um delineamento inteiramente casualizado. Os níveis de incidência de frutos danificados por lesmas nas parcelas foram significativos em baixas populações (0,1 e 2 ind./ armadilhas) aumentando para 40\%. Perdas monetárias de lesmas podem exceder 50.000.000 COP / ha com taxas de até 2 lesmas / armadilha.
\end{abstract}

Palavras-chave: moluscos, estimação, danos, colheita 


\section{INTRODUCCIÓN:}

Las babosas pertenecen a la clase Gastropoda, subclase Pulmonata y son de gran importancia agrícola, se pueden desarrollar dentro de un amplio rango de temperatura (Cañedo, Alfaro \& Kroschel, 2011); teniendo gran preferencia para hacer actividad por los climas templados (Serre, 2005) y tienen gran preferencia por humedades relativas altas (Herrera \& Castellanos, 2013) y atacan a varias hortalizas incluyendo la fresa (Nodarse, Castellanos, Pérez y Becerra, 2015).

Los moluscos y dentro de ellos, las babosas pueden causar pérdidas económicas importantes a los cultivos a libre exposición o bajo cubierta, debido a que disminuyen los bajas en el rendimiento por la reducción del área foliar y en algunas ocasiones generan daños en los frutos y en ocasiones las producciones agrícolas pueden verse severamente afectadas por sus voraces hábitos alimenticios. Esto se puede evidenciar también, en fincas de agricultura urbana, las cuales tienen gran impacto económico (Vázquez, Gonzálvez, Lauzardo, García, Simonetti y Ramírez, 2005). Las babosas pueden llegar a ocasionar bajas en el rendimiento de los cultivos de organopónicos a medida que aumenta la población de los individuos (Becerra, 2016).

Martínez, Bohórquez y Acosta (1994) informan cinco grupos de babosas pertenecientes a los géneros Arion, Milax y Deroceras causando daños en la Empresa Jardines de Colombia en Cundinamarca. Constantino et al. (2010) reportan dos especies de moluscos atacando plantaciones de café: Colosius pulcher (Colosi) y Sarasinula plebeia (Fischer).

En el cultivo de la papa se informan Deroceras reticulatum (Müller) babosa pequeña; Limax marginatus (Müller) babosa parda rayada, Milax gagates (Draparnaud) babosa gris y Veronicella spp. babosa plana (ICA, 2011), mientras que para las hortalizas se informa a Deroceras reticulatum, a Limax marginatus y a. M. gagates (ICA, 2012).

Muchas recomendaciones se dan por varios autores entre ellos Martínez et al. (1994) sobre la necesidad de profundizar en la taxonomía, ecología, los métodos de manejo, el control biológico y la evaluación de los daños de las babosas plagas, sin embargo, las publicaciones son escasas y casi nulas sobre las evaluaciones de pérdidas económicas a causas de estos moluscos.

En las condiciones de Pamplona Norte de Santander gran parte de los cultivos presentan daños a causa de las babosas, sin embargo los estudios publicados se reducen a los Hernández et al. (2015) los de Méndez y Castellanos, (2017) que evaluaron la cal agrícola y la tierra de diatomeas contra el caracol del jardín Helix aspersa Muller en condiciones de laboratorio y a los de Méndez y Castellanos, 2019) que evaluaron esos productos alternativos contra Arionidos y Agrolimaceos en condiciones de laboratorio y campo.

Peñaranda y Molina, (2011) en una investigación desarrollada en la Vereda Monteadentro, Pamplona, Norte de Santander recomendaron aumentar las investigaciones científicas sobre los agentes fitosanitarios con el objetivo de disminuir el 
excesivo empleo de agroquímicos, que al final contaminan el río Pamplonita principal colector de las aguas fluviales de esa cuenca (Ortega,1916). Se pudo constatar que no se han realizado investigaciones relacionadas con las pérdidas económicas de las babosas en los cultivos bajo estas condiciones, que pudieran aportar el umbral económico para acometer las medidas de control y hacer un uso racional de los productos químicos e impactar lo menos posible al medio ambiente. En el contexto económico el trabajo toma como referencias en tres contextos desde el análisis económico de Colombia plantado por Caballero Tovío Alfaro (2016); el riesgo financiero que genera pérdidas económicas en la empresas en Colombia presentadas por Castro Albarracín Muñóz, García Arenas y García (2017) y Bastos, Mogrovejo, y Gómez (2015).

El objetivo de la presente investigación fue estimar las pérdidas económicas causadas por las babosas en fresa (Fragaria $\times$ ananassa, Duch) bajo las condiciones de Pamplona, Norte de Santander.

\section{METODOLOGÍA:}

Para la determinación de las pérdidas de cosecha y económicas por las babosas en fresa bajo las condiciones de Pamplona se condujo un experimento en un campo de un predio cultivado con la variedad Albión en la vereda el Rosal de este municipio con coordenadas norte 72149,6 y oeste $-724014,7$ a 2624 m.s.n.m. El lote presentaba una población de cuarenta mil (40000) plantas sembradas en camas de 1,20 m de ancho con doble surco a tresbolillo a treinta por treinta centímetros entre plantas y surcos $(30 \mathrm{~cm} \times 30 \mathrm{~cm})$ y una edad de seis (6) meses de producción.

El área experimental neta contó con quinientos metros cuadrados $\left(500 \mathrm{~m}^{2}\right)$ distribuidas en todo el lote, conformada por cinco (5) parcelas con un área experimental física de cien metros cuadrados $\left(100 \mathrm{~m}^{2}\right)$ para cada tratamiento, las cuales se dispusieron con una separación aproximada de diez metros $(10 \mathrm{~m})$ entre ellas. Se condujo el experimento en un diseño completamente aleatorizado. Las parcelas se subdividieron en veinticinco metros cuadrados $\left(25 \mathrm{~m}^{2}\right)$ de área experimental para contar con cuatro repeticiones aleatorias; contando todas con un mismo manejo agronómico.

Basado en la distribución espacial natural de las babosas en el campo completo teniendo en cuenta los cultivos y áreas naturales colindantes según recomendaciones de Serre (2005) y las sugerencias del agricultor en cuanto a los niveles de incidencia de menor a mayor de las poblaciones de babosas se determinó la ubicación de las parcelas para cada tratamiento. Para conocer la distribución espacial de las babosas en el campo experimental se ubicaron una semana antes de iniciar el experimento 30 trampas de refugio construidas de cartón con un de $25 \times 25 \mathrm{~cm}$ sujetadas con una cabilla metálica de $0,30 \mathrm{~cm}$ de diámetro y $40 \mathrm{~cm}$ de longitud en forma de "L" para evitar que fueran movidas por el viento. 
Posterior al conocimiento de la distribución espacial de las babosas en el campo, se decidieron los siguientes tratamientos.

\begin{tabular}{lll}
\hline Tratamiento & \multicolumn{1}{c}{$\begin{array}{c}\text { Parcela } \\
\text { correspondiente }\end{array}$} & \multicolumn{1}{c}{$\begin{array}{c}\text { Individuos por } \\
\text { tratamiento }\end{array}$} \\
\hline Tratamiento & Parcela 3 & $\begin{array}{l}\text { Presencia de } 0 \\
\text { babosas }\end{array}$ \\
1 & & $\begin{array}{l}\text { Presencia de 0,1 a 2 } \\
\text { individuos/trampa }\end{array}$ \\
Tratamiento & Parcela 2 & Presencia de 2,1 a 4 \\
2 & & individuos/trampa \\
Tratamiento & Parcela 1 & Presencia de 4,1 a 6 \\
3 & & individuos/ trampa \\
Tratamiento & Parcela 4 & Presencia mayor a \\
4 & & 6,1 \\
Tratamiento & Parcela 5 & individuos/trampa \\
\hline
\end{tabular}

El tratamiento 1 en la parcela 3, se mantuvo con cero (0) individuos por trampa, mediante la aplicación de un molusquicida granulado de cebo tóxico de categoría toxicológica III, MATABABOSA® Metaldehído $(2,4,6$, 8 tetramatil-1, 3, 7-tetraoxa ciclo octano), solamente a la parcela de dicho tratamiento (SUPERABONO LTDA., 2013). Adicionalmente se colocó un cordón con cal agrícola alrededor de la parcela, el cual se reponía semanalmente, para evitar que entraran babosas de las áreas colindantes.

Las poblaciones de babosas en cada parcela pequeña $\left(25 \mathrm{~m}^{2}\right)$ se monitorearon dos veces por semana mediante la ubicación de 6 trampas de refugio como las descritas anteriormente. De esta forma se mantuvieron las poblaciones de las babosas en las parcelas experimentales según los tratamientos prestablecidos compensando las poblaciones dos veces /semana. El experimento se desarrolló durante cinco (5) semanas.

La ubicación espacial de los tratamientos quedó de la siguiente forma (Figura 1):

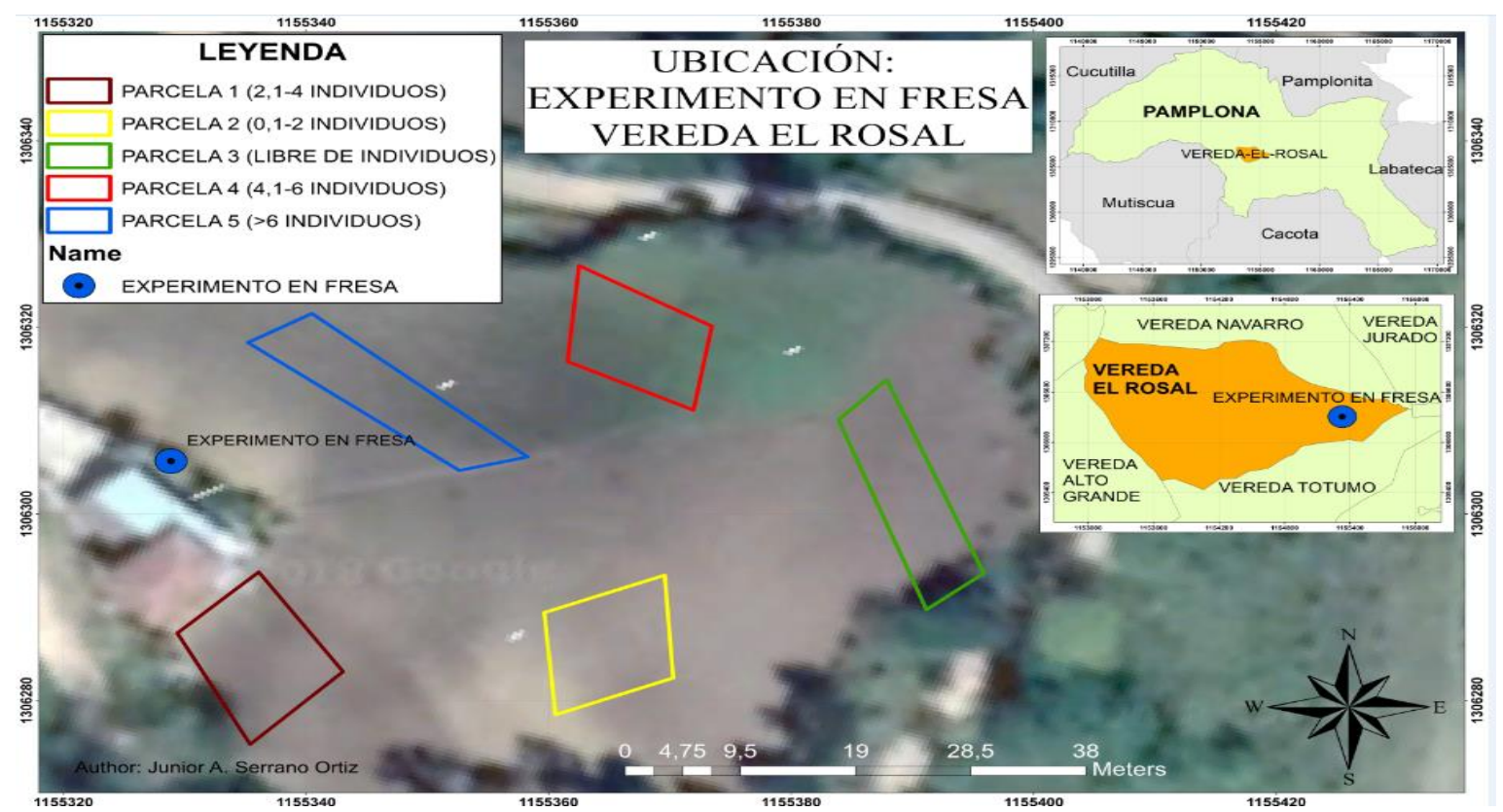

Figura 1. Diagrama del diseño experimental para la pérdida económica en el cultivo de fresa en la vereda EL Rosal, elaborada mediante el programa ArcGIS 10.5, basada en la base de datos del IGAC (www.igac.gov.co). 
regresión para buscar modelos matemáticos entre la perdida monetaria como variables dependientes en función de la población de babosas como variable independiente. Para todos los análisis estadísticos se empleó el paquete SPSS versión 21 para Windows (IBM, 2012).

\section{RESULTADOS Y DISCUSIÓN:} recolectaron doscientos (200) frutos al azar en las cinco parcelas grandes, para su posterior pesaje calculando el peso promedio de un fruto; este procedimiento se realizó cinco (5) veces para calcular el peso promedio del fruto en el terreno. También se contaron los frutos cosechados en las 25 plantas en cada cosecha. A partir de esta información y el porcentaje de incidencia de los frutos dañados por planta se estimó la perdida en kg / planta parcela por tratamiento.

La pérdida económica de cada tratamiento en el experimento se estimó a partir de los precios de comercialización establecidos oficialmente, siendo de tres mil pesos colombianos el kilogramo de fresa (3000 COP/kg) (DANE, 2018) y la pérdida en rendimiento evidenciada semana a semana.

Los datos obtenidos de incidencia del daño por parcela se transformaron en arc sen $\sqrt{ } \% / 100$ y se sometieron a un análisis de varianza para un factor, considerando las parcelas pequeñas como repeticiones, una vez comprobado la supuesta de normalidad por la prueba de Kolmogorov Smirnov. Se realizó el análisis de
Aunque fueron las babosas predominantes el área del experimento pertenecían a Deroceras spp. y Arion spp. también estaban presentes en menor medida Milax sp. En todos los momentos de evaluación los porcentajes de incidencia de frutos afectados por babosas en las parcelas con diferentes niveles poblacionales difirieron del tratamiento con cero poblaciones de babosas. Los niveles de incidencia de frutos dañados por babosas en las parcelas no tratadas variaron en los diferentes momentos entre 13,9\% para poblaciones de babosas entre 0,1 y 2 ind./trampas y $40,51 \%$ cuando las poblaciones se mantenían por encima de 6 ind./trampas (Tabla 1), lo que da una idea de la afectación que puede ocurrir en el campo cuando la población de babosas es alta. 
Tabla 1. Porcentaje de incidencia de daño por babosas en cada momento, en el experimento en la vereda el Rosal.

\begin{tabular}{cccccc}
\hline & \multicolumn{5}{c}{ Incidencia de frutos dañados por babosas (\%) } \\
\hline Tratamiento & M1 & M2 & M3 & M4 & M5 \\
\hline Tratamiento 1 (Libre de & $0 \mathrm{c}$ & $0 \mathrm{c}$ & $0 \mathrm{~d}$ & $0 \mathrm{~d}$ & $0 \mathrm{~d}$ \\
individuos) & & & & & \\
Tratamiento 2 (0.1-2 & $16,14 \mathrm{~b}$ & $16,49 \mathrm{~b}$ & $13,44 \mathrm{c}$ & $13,91 \mathrm{c}$ & $18,46 \mathrm{c}$ \\
$\quad$ Individuos) & & & & & \\
Tratamiento 3 (2.1-4 & $17,1 \mathrm{~b}$ & $17,54 \mathrm{~b}$ & $16,11 \mathrm{c}$ & $20,47 \mathrm{c}$ & $24,79 \mathrm{~b}$ \\
$\quad$ Individuos) & & & & & \\
Tratamiento 4 (4.1-6 & $33,73 \mathrm{a}$ & $30,9 \mathrm{a}$ & $29,22 \mathrm{~b}$ & $25,4 \mathrm{~b}$ & $26,19 \mathrm{~b}$ \\
Individuos) & & & & \\
Tratamiento 5 (> 6.1 Individuos) & $36,9 \mathrm{a}$ & $38,45 \mathrm{a}$ & $39,56 \mathrm{a}$ & $40,51 \mathrm{a}$ & $35,49 \mathrm{a}$ \\
C. V. (\%) & 16,84 & 23,39 & 20,93 & 23,92 & 11,79 \\
Error típico* & 1,75 & 2,42 & 2,06 & 2,4 & 1,24 \\
\hline
\end{tabular}

${ }^{*}$ Letras desiguales en las columnas difieren para $\mathrm{P} \leq 0,05$ por la prueba de Tukey. M= Momento.

Fuente: Elaboración propia del autor

En las dos primeras semanas de muestreo los valores más altos de incidencia de perjuicios en fruto se presentaron en los tratamientos 4 y 5 con diferencia de los tratamientos con poblaciones de 0,1 a 2 y de 2 a 4 babosas/trampa, difiriendo todos del testigo. En las evaluaciones de las semanas 3 y 4 el tratamiento 5 con índices de más de 6 babosas/trampas presentó los mayores niveles de incidencia, con diferencia con el resto de los tratamientos que tenían babosas, mientras que el tratamiento 4 difería de este y del 2 y 3.

En la última semana el tratamiento 5 mantuvo el mayor nivel de incidencia, le siguieron el 4 y 3 que no se diferenciaron entre sí y el 1 y 2 que se diferenciaban del testigo y también del resto a pesar de tener solo índices entre 0,1 y 2 babosas por trampa. 
La pérdida en peso de frutos por planta obtenida en el experimento para los tratamientos con diferentes niveles poblacionales osciló entre 0,30 y $0,70 \mathrm{~kg} / \mathrm{planta}$ (Tabla 2):

Tabla 2. Pérdida en peso (kg/planta) en cada momento ocasionado por babosas, en el experimento en la vereda el Rosal.

\begin{tabular}{|c|c|c|c|c|c|c|}
\hline Tratamiento & & M1 & M2 & M3 & M4 & M5 \\
\hline $\begin{array}{l}\text { Tratamiento } 1 \\
\text { individuos) }\end{array}$ & (Libre de & 0,00 & 0,00 & 0,00 & 0,00 & 0,00 \\
\hline $\begin{array}{l}\text { Tratamiento } \\
\text { Individuos) }\end{array}$ & $2 \quad(0.1-2$ & 0,30 & 0,34 & 0,30 & 0,39 & 0,34 \\
\hline $\begin{array}{l}\text { Tratamiento } \\
\text { Individuos) }\end{array}$ & $3 \quad(2.1-4$ & 0,32 & 0,31 & 0,26 & 0,26 & 0,47 \\
\hline $\begin{array}{l}\text { Tratamiento } \\
\text { Individuos) }\end{array}$ & $4 \quad(4.1-6$ & 0,65 & 0,59 & 0,56 & 0,48 & 0,50 \\
\hline $\begin{array}{l}\text { Tratamiento } \\
\text { Individuos) }\end{array}$ & $\begin{array}{lll}5 & > & \\
& & \end{array}$ & 0,70 & 0,73 & 0,71 & 0,77 & 0,67 \\
\hline
\end{tabular}

Nota: M= Momento. Datos obtenidos del procesamiento de los datos de las visitas a campo.

Fuente: Elaboración propia del autor

Se observa que las pérdidas en peso se incrementan en la medida que aumentan las poblaciones en todas las semanas de observación. A partir de la estimación que un fruto de fresa pesaba aproximadamente $0,019 \mathrm{~kg}$, en el tratamiento 4 con una población de 4,1 a 6 individuos por planta podrían haber afectados aproximadamente 0,5 kg/planta.

Los datos de las pérdidas monetarias ocasionadas por los moluscos en el predio durante el tiempo transcurrido del experimento se expresan en la Tabla 3.

Tabla 3. Pérdida monetaria (COP/planta) en cada momento ocasionado por babosas, en el experimento en la vereda el Rosal.

\begin{tabular}{llllll}
\hline Tratamiento & M1 & M2 & M3 & M4 & M5 \\
\hline Tratamiento 1 (Libre de individuos) & 0,00 & 0,00 & 0,00 & 0,00 & 0,00 \\
Tratamiento 2 (0.1-2 Individuos) & 910,82 & 1016,56 & 913,26 & 1168,32 & 1033,33 \\
Tratamiento 3 (2.1-4 Individuos) & 964,09 & 931,58 & 769,05 & 782,35 & 1399,09 \\
Tratamiento 4 (4.1-6 Individuos) & 1945,67 & 1782,72 & 1668,92 & 1443,35 & 1493,95 \\
Tratamiento 5 (> 6.1 Individuos) & 2087,91 & 2204,94 & 2125,82 & 2305,91 & 2019,19 \\
\hline
\end{tabular}

Nota: $\mathrm{M}=$ Momento. Datos obtenidos del procesamiento de los datos de las visitas a campo.

Fuente: Elaboración propia del autor 
De esta se infiere que con poblaciones entre 2 y 6 babosas/trampa se pueden perder entre 1000 y 1900 COP (pesos colombianos) por planta, considerando que el precio en el mercado del kilogramo de fresa se encuentraba a 3000 COP (DANE, 2018). Si se tiene en cuenta una densidad de plantación óptima de 51500 plantas /ha (Angulo, 2009) similar a la que se emplea en Pamplona se pudieran perder entre 51000000 y 77 $850000 \mathrm{COP} / \mathrm{ha}$ bajo estas condiciones semanalmente cuando la plantación de fresa variedad Albión está em plena producción.

El modelo de regresión lineal obtenido muestra un $\mathrm{R}^{2}$ equivalente a 0,83 , es decir que la ecuación tiene un $83 \%$ de efectividad para predecir la perdida monetaria en función de la incidencia de las babosas en un campo de fresa de la variedad Albión en las condiciones de Pamplona (Figura 2).

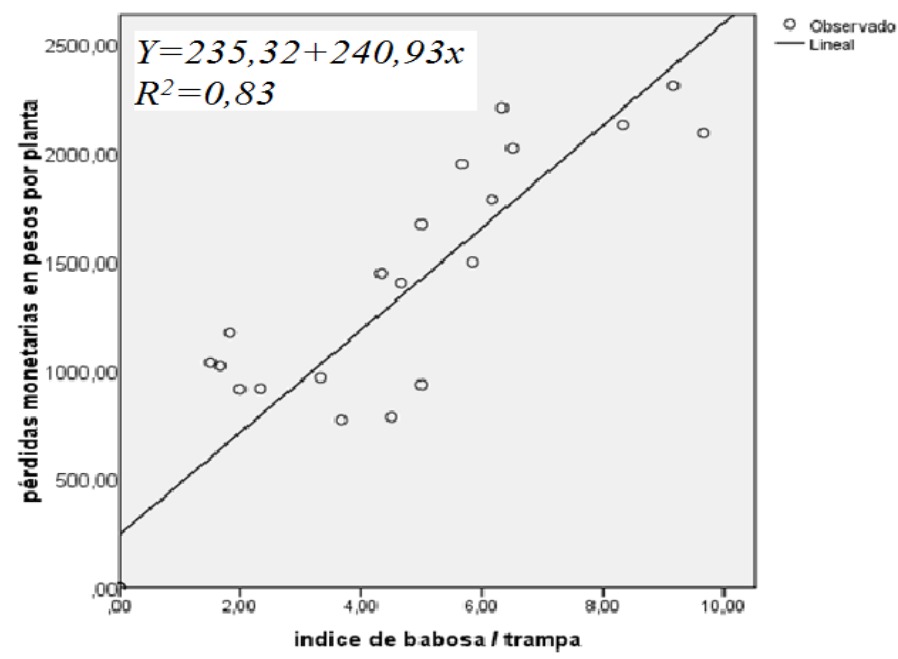

Figura 2. Modelo matemático de regresión lineal de la pérdida monetaria en pesos colombianos por planta en dependencia de la cantidad de babosas por trampa. $\mathrm{R}^{2}=$ Coeficiente de determinación.

Fuente: Elaboración propia del autor
Becerra (2016) determinó porcentajes de pérdidas en rendimientos en remolacha de hasta $25,74 \%$ y en lechuga de $28,8 \%$ en lechuga con poblaciones de 4 moluscos $/ \mathrm{m}^{2}$, perdidas inferiores a los determinados en el presente estudio lo cual es atribuible a que el producto comercializable de la remolacha no es afectado por las babosas y que de la lechuga puede beneficiarse y comercializarse la parte no afectada por las babosas, mientras que en la fresa se afecta el fruto comercializable. Al comparar los resultados obtenidos con otros experimentos como el caso del cultivo de arroz en Perú, se consideraron daños importantes a partir de poblaciones de 6,4 individuos por macolla (Jiménez, 2004) similar a los obtenido en presente trabajo.

Caso diferente se informa en el cultivo del café (Constantino et al., 2010), quien consideró que las poblaciones hacen daño en este cultivo cuando alcanzan valores de 15 individuos/planta.La alta incidencia de las babosas en la fresa en las condiciones de Pamplona infiere continuar en la búsqueda de alternativas de control con bajo nivel de riesgo para la salud del consumidor ya que es precisamente el fruto el que es preferido por estos moluscos.Se hace necesario continuar las investigaciones para determinar los porcentajes de pérdidas de rendimiento y monetarias que ocurren en otros cultivos importantes y con alta productividad en Pamplona como la papa amarilla, la alverja y las hortalizas (Gualdrón, Maldonado, Espitia y García, 2017) y que son afectados por las babosas (ICA, 2011; ICA, 2012). 


\section{CONCLUSIONES:}

Los niveles de incidencia de frutos dañados por babosas en las parcelas fueron significativos a bajas poblaciones ( 0,1 y 2 ind./trampa) incrementándose con el aumento de los niveles poblacionales hasta $40 \%$, cuando los índices sobrepasaron los 6 ind./trampa. El modelo matemático $Y=235,32+240,93 x$, permite estimar las pérdidas monetarias en pesos colombianos para la fresa variedad Albión en el periodo de cosecha en función de las poblaciones de las babosas en las trampas de refugio.

Las pérdidas monetarias por babosas en fresa bajo las condiciones de Pamplona son cuantiosas pudiendo superar las $50000000 \mathrm{COP} / \mathrm{ha}$ con índices de hasta 2 babosas/trampa, lo cual puede incrementarse linealmente en la medida que aumentan las poblaciones de estos moluscos.

\section{REFERENCIAS:}

Angulo, R. (2009). Fresa Fragaria annassa. Bayer CropScience S. A. Bogota. Colombia. 48p.

Albarracín Muñóz, M., García Arenas, L., \& García, C. (2017). Riesgo financiero: una aproximación cualitativa al interior de las mipymes en

Colombia. Aglala, 8(1), 139-160.

https://doi.org/https://doi.org/10.22519/22157360.102 $\underline{9}$
Becerra, F. E. J. (2016). Nivel de daño económico de moluscos plagas en cultivos de Lactuca sativa L. y Beta vulgaris L. del organopónico T-15. Tesis de grado. Universidad Cienfuegos.

Bastos, L., Mogrovejo, J., \& Gómez, L. (2015). Experiencias del Branding como estrategia para el posicionamiento en el mercado internacional. Revista Face, facultad deficiencias económicas y empresariales, 15(1), 3.

Caballero Tovío, A., \& Castro Alfaro, A. (2016). Análisis del comportamiento de las variables que determinan el circuito económico en Colombia. Aglala, $\quad 7(1), \quad$ 94-121. doi:10.22519/22157360.975

Cañedo, V., Alfaro, A. \& Kroschel, J. (2011). Manejo integrado de plagas de insectos en hortalizas. Principios y referencias técnicas para la Sierra Central de Perú. Centro Internacional de la Papa (CIP), Lima, Perú. Recuperado el 25 de octubre de 2018. Sitio web: http://cipotato.org/wpcontent/uploads/2014/08/005739.pdf

Constantino, L.M., Gomes, S. \& Benavides, P. (2010). Descripción y daños causados por las babosas Colosius pulcher y Sarasinula plebeia en el cultivo de café en Colombia. Centro Nacional de Investigación de Café (Cenicafé). Chinchiná, Caldas, Colombia. 
DANE. (2018). Boletín semanal de precios mayoristas. Sistema de Información de Precios y Abastecimiento del Sector Agropecuario (SIPSA). Recuperado el 07 de enero de 2019. Sitio web: https://www.dane.gov.co/index.php/estadistic as-por-tema/agropecuario/sistema-deinformacion-de-precios-sipsa

Gualdrón, G. C. A., Maldonado, G. B. A, Espitia, V. D. R. \& García, S. J. N. (2017). Aproximación al caso de desarrollo local de la zona rural del municipio de Pamplona. Universidad de Pamplona, Norte de Santander, Colombia. FACE: Facultad de Ciencias Económicas y Empresariales. $17,2: 142-154$

Hernández, B. L., Guerrero, N. M., \& Sierra, M. P. (2015). Determinación de los daños en babosas (Arion distinctus) causado por la tierra diatomea a diferentes concentraciones bajo condiciones de laboratorio en el ISER, Pamplona. Revista Distancia al Día, 1(1), 1-9. http://www.iser.edu.co/iser/hermesoft/portall G/home_1/recursos/documentos_generales/ 16102015/inv_revista_al_dia_mayo_2015.pd $f$

Herrera, N. \& Castellanos, L. (2013). Informe sobre la incidencia de moluscos plaga en organopónicos del municipio de Cienfuegos, Centro Agrícola, 40(1):89-90.

IBM. (2012). IBM SPSS Statistics for Windows, Versión 21.0. New York: IBM Corp. ISBN-13: 9780205985517. Recuperado de https://www.ibm.com/analytics/us/en/technol ogy/spss/

ICA. (2011). Manejo fitosanitario del cultivo de la papa (Solanum tuberosum subsp. andigena y $S$. phureja). Bogotá D.C. Colombia.

ICA. (2012). Manejo fitosanitario del cultivo de hortalizas. Bogotá D.C. Colombia.

Jiménez, G. L. E. (2004). Determinación del daño de babosa (Gasteropoda: Limacidae) en el cultivo de arroz (Oryza sativa L.), variedad Capinona, bajo condiciones semicontroladas en el Bajo Mayo-Tarapoto. Universidad Nacional de San Martín. Tarapoto, Perú.

Martínez, J. W., Bohórquez, S. L. \& Acosta, A. (1994). Determinación taxonómica de cinco grupos de babosas y estudio del ciclo de vida del grupo predominante en un cultivo comercial de alstroemeria de Madrid-Cundinamarca. Agronomía colombiana, $X I$ 1;53-61.

Méndez, A.C., \& Castellanos, L. (2017). Eficacia de la tierra de diatomeas contra Helix aspersa en condiciones in vitro en Pamplona, Norte de Santander. Journal of Negative \& No Positive Results, 2(12), 59-666. 
Méndez, A.C., \& Castellanos, L. (2019). Eficacia de la tierra de diatomeas y la cal sobre ariónidos y agriolimácidos. Revista Corpoica. Ciencia Tecnol Agropecuaria, 20(3): 579-593, 2019. 0122-8706,

http://revistacta.agrosavia.co/index.php/revist $\underline{a}$

Mosquera, J., \& Reyes, C. M. (2013). La valoración y recuperación de saberes como instrumento para la construcción de territorialidad y el desarrollo endógeno. FACE: Revista de la Facultad de Ciencias Económicas y Empresariales, 13(1), 151-170. Recuperado de:

http://revistas.unipamplona.edu.colojs vicein ves/index.php/FACE/article/view/963.

Nodarse, M. Castellanos, L. Pérez, A., \& Becerra E.J. (2015). Eficacia de los jugos de Furcraea hexapétala y Agave brittoniana para el control de moluscos plagas en acelga en organopónicos Agroecosistemas.3, 2: 488493.

Ortega, J. Y. (2016). Estructura ecológica principal de la cuenca del Río Pamplonita. Revista Ambiental Agua, Aire y Suelo. ISSN 19009178. Volumen (7), Numero (1). DOI: https://doi.org/10.24054/19009178.v1.n $\underline{1.2016 .3258}$
Peñaranda, G., Molina, D.Y. (2011). La producción de arveja (Pisum sativum) en la vereda Monteadentro, provincia de Pamplona, Norte de Santander. Revista FACE. 8(11):43-56.

Serre, M. (2005). Manejo de babosas en el cultivo de girasol en siembra directa. Departamento de Agronomía, Pioneer Argentina S.A. Recuperado el 13 de octubre de 2018.

SUPERABONO LTDA. (2013). Hoja De Seguridad MATABABOSA®. Departamento Ambiental. Colombia.

Vázquez, L., Gonzálvez, E., Lauzardo, J., García, T., Simonetti, J. \& Ramírez, R. (2005). Manejo agroecológico de plagas en fincas de la agricultura urbana (MAPFAU). Ediciones CIDISAV. La Habana, Cuba. 